\title{
Effects of static stretching duration on muscle stiffness and blood flow in the rectus femoris in adolescents
}

\author{
Emine Caliskan', Orkun Akkoc², Zuhal Bayramoglu³, Omer Batin Gozubuyuk ${ }^{4}$, Doga Kural'2, \\ Sena Azamat ${ }^{3}$, Ibrahim Adaletli ${ }^{3}$
}

${ }^{1}$ Department of Pediatric Radiology, Seyhan State Hospital, Adana, ${ }^{2}$ Department of Moving and Training Science, Faculty of Sports Science, Istanbul University, Istanbul, ${ }^{3}$ Department of Pediatric Radiology, Istanbul University Faculty of Medicine, Istanbul, ${ }^{4}$ Department of Sports Medicine, Istanbul University Faculty of Medicine, Istanbul, Turkey

\begin{abstract}
Aims: To compare the effects of 2 and 5 min of passive static stretching (SS) on stiffness and blood flow in the rectus femoris in adolescent athletes using shear wave elastography (SWE) and superb microvascular imaging (SMI). Material and methods: This prospective study included 20 male athletes with median age of 14.5 (12.5-16.5) years. The subjects were divided into two groups based on the SS duration as follows: $2 \min (n=10)$ and $5 \min (n=10)$. At rest and after 2 and 5 min of SS, stiffness and blood flow values were compared in the rectus femoris for each group. Inter-operator reliability was also analysed. Results: There was no significant difference between resting and 2 min of SS in terms of stiffness. The stiffness values decreased significantly from resting to $5 \mathrm{~min}$ of SS. The blood flow increased significantly from resting to 2 and $5 \mathrm{~min}$ of SS. Inter-operator reliability was moderate to perfect for SWE and SMI measurements (ICC: 0.52-0.83). Conclusions: SWE and SMI can be used to acquire reliable quantitative data about muscle stiffness and blood flow in adolescents. While stiffness parameters significantly decreased from resting after only $5 \mathrm{~min}$, blood flow significantly increased both after 2 and 5 min. For physical rehabilitation protocols, 5 min of SS may be chosen to reduce stiffness. For competitions, 2 min of SS may be sufficient for warm-up exercise because it increases the blood flow optimally. Five min of SS may be preferred for the cool-down exercise to enhance recovery.
\end{abstract}

Keywords: blood flow; muscle stiffness; shear wave elastography; static stretching; superb microvascular imaging

\section{Introduction}

Cramps, spasms and traumatic damage cause microstructural disruptions in the muscle tissue [1]. They alter the healthy condition and trigger muscle stiffening. Muscle stretching increases the speed of recovery from cramps [2] and it is popular among health therapists and athletic trainers because it reduces muscle stiffness and the incidence of muscle injury and increases flexibility [3-6].

Received 31.12.2018 Accepted 24.02.2019

Med Ultrason

2019, Vol. 21, No 2, 136-143

Corresponding author: Emine Caliskan

Haci Omer Sabanci Street, Seyhan,

Adana, Turkey, 11050

ORCID: 0000-0001-9869-1396

E-mail:eminecaliskanrad@gmail.com
Muscle stretching is widely performed as a warm-up or cool-down exercises for subjects undergoing physical activity. Stretching may be applied with different techniques including mainly static (active tensioning, passive, isometric), ballistic, dynamic, and proprioceptive neuromuscular facilitation (PNF). Static stretching (SS) is a technique that increases flexibility by the greatest amount. Passive SS aims to preserve joint and muscle stretching through the effect of an external force, such as a partner's push, wall, floor or machine, which is applied to attain and hold the end position [7]. It may be applied for short (2-3 min or less) and longer (5-10 min or longer) durations [8].

Skeletal muscle stretching is accepted as an adaptation of low intensity exercise and it has significant effects on general health care and vascularity [9]. The microcirculation in normal skeletal muscle consists of a highlyorganized network of arterioles, capillaries and venules 
that are structurally arranged to optimize oxygen transport. Voluntary or involuntary changes in local blood flow physically and clinically affect the muscle structure. Increasing blood flow provides a greater amount of oxygen to the muscles and the opportunity to produce higher levels of physical performance [10]. As muscles are stretched, blood flow and oxygen delivery are compromised and consequently muscle function is impaired. It is established that a robust angiogenesis and endotheliumdependent vasodilatation response can occur following an ischemic injury to the skeletal muscle in the acute or chronic recovery period $[11,12]$. This neovascularization response, together with the opening of collateral vessels, can restore blood flow to the otherwise compromised muscle. Hotta et al reported that 4 weeks of daily muscle stretching enhanced endothelium-dependent vasodilatation of skeletal muscle resistance arterioles in aged rats [12]. However, no published data provide sufficiently quantitative information regarding acute changes in muscular blood flow after stretching in humans.

Shear wave elastography (SWE) is a new imaging technique that provides information regarding tissue stiffness by a higher intensity pulse that is transmitted into the body to produce shear waves, which extend laterally from the target tissue and are then tracked with low-intensity pulses to find the shear velocity related to Young's modulus. Tissue stiffness measurements may be beneficial for the identification of muscles as well as a variety of organ disorders [13-15]. Elastography studies investigating the acute effects of stretching (ranging from 2-10 min duration) on the adult's triceps surae, hamstring and shoulder muscles have previously been performed [16-21].

Superb microvascular imaging (SMI) is an advanced Doppler method. It provides a great deal of data regarding the intra-lesion vascular network. Compared to conventional Doppler techniques such as power Doppler and color Doppler, it uses a higher frame rate. Tiny vessels can be clearly detected using SMI by suppressing scattering and demonstrating signals of low-velocity flows based on the ability to distinguish motion artifacts from slow-velocity signals [22].

The pediatric population should be considered separately from adults because the pediatric population is exposed to many hormonal and biological changes especially in the transition from childhood (under 10 years) to adolescence (above 10 years), unlike adults. According to elastography studies on adults, stretching reduces muscle stiffness [16-21]. However, no work evaluating the effect of SS duration in adolescents exists in the literature. Studies related to muscle blood flow used conventional Doppler, micro-CT analysis, gamma scintillation counter or experimental model methods $[12,23]$. These studies have concluded that there is a reduction in local blood flow during stretching, with an acute post-stretch hyperemic response after stretching and a vascularity increase in the chronic period. Similarly, the acute effect of stretching on blood flow using SMI has not been previously evaluated. Hypothetically, determination of the acute effects of SS duration on stiffness and blood flow will be beneficial for physical rehabilitation, to prevent muscle injury and to prepare for athletic performance in adolescents. We also hypothesized that we can quantitatively show the acute increase in muscle blood flow using SMI after stretching.

Therefore, we used SWE and SMI to determine the effects of passive SS durations ( 2 and 5 minutes SS) on muscle stiffness and blood flow. Our target population and muscle were adolescent male athletes and the rectus femoris $(\mathrm{RF})$, respectively. We also investigated interoperator reliability for SWE and SMI.

\section{Material and methods}

\section{Subjects}

Twenty male athletes with the median age of 14.5 (12.5-16.5) years volunteered to participate in this prospective study. All of them had played in young basketball leagues for at least 5 years of duration. They had no history of traumatic or non-traumatic lower extremity injuries in the last 6 months. Subjects with medical history, chronic drug use, rheumatic, systemic and/or connective tissue disorders were not included in the study. The study was conducted with ethics approval from the local Ethics Committee, and signed informed consent was obtained from the parents in all cases.

\section{Study design}

Muscle stiffness and blood flow were measured in the supine position with the knee extended for RF measurements. The participants were informed about the procedure and asked to stay as relaxed as possible during each measurement. Subjects were randomly divided into two groups, each including ten participants. In group A, outcome measures were assessed before (pre-) and after (post-) 2 min of SS. In group B, measures were assessed before (pre-) and after (post-) 5 min of SS. All subjects were examined in the same day by two pediatric radiologists with over 5 years of SWE and 2 years of SMI experience. Each radiologist performed the acquisition of SWE and SMI in the same position and was blinded to the data gathered by the other operator. Statistical comparisons of stiffness and blood flow values were made between the groups. Inter-operator reliability was also analysed. 


\section{Shear Wave Elastography and Superb \\ Microvascular Imaging Techniques}

Measurements were examined using an Aplio 500 Platinum ultrasound device (Canon Medical Systems, Japan) with a high-frequency linear transducer (frequency range, $7.2-14 \mathrm{MHz}$ ). The entire probe surface was covered with ultrasonic gel that was 3-4 mm thick to ensure optimal image quality and to minimize the transducer pressure on the skin.

The measurement location was determined based on previous studies [24]. The same location was used for both SWE and SMI examinations. RF was examined at the midpoint between the lateral epicondyle of the femur, and the femoral greater trochanter. The orientation of the transducer for the SWE technique was referenced from previous studies to achieve accurate and reliable measurements [25]. The muscle was located with the transducer oriented axially. Then, the longitudinally oriented transducer was turned perpendicular to the plane to measure the stiffness. Same transducer orientation was used for the SMI technique to measure blood flow.

While obtaining the images, pressure was not applied to the probe and care was taken that the operator's hand was motionless. In split-screen mode, 2D-SWE map (left side) and quality mode (right side) were examined. The quality mode, which is identified as the propagation mode (arrival time contour), is a mode in which reliable data is obtained when the lines are parallel and smooth, and the increase in distance between lines is parallel to the increase in stiffness. The speed of ultrasound waves was either measured in kilopascal $(\mathrm{kPa})$ for elasticity and meters $/ \mathrm{second}(\mathrm{m} / \mathrm{s})$ for velocity; within seconds, the result was displayed on the ultrasound screen. During stabilization of SWE images for 5 seconds, SWE images were frozen and saved. The elasticity range was set to $0-80 \mathrm{kPa}$ and the velocity range was set to $0-8 \mathrm{~m} / \mathrm{s}$ on a standardized "musculoskeletal preset mode". Subsequently, a $5 \mathrm{~mm}$ diameter "region of interest (ROI)" was used to take measurements at three different points with three repeated acquisitions in the longitudinal view (fig 1). Mean elasticity and velocity values were automatically calculated by averaging nine values.

SMI investigation used a frame rate set at $>50 \mathrm{~Hz}$ and the pulse repetition frequency was set at $220-234 \mathrm{~Hz}$. The color gain was set to $30-40$ decibels to suppress background artifacts and SMI imaging used the color mode. The vascularity index (VI [\%]) measurement method was used to quantify blood flow. In the color mode, a rectangular ROI was manually drawn on a fixed window with $15 \times 10 \mathrm{~mm}$ dimensions. Within the ROI, the proportion of color pixels in the whole area was automatically

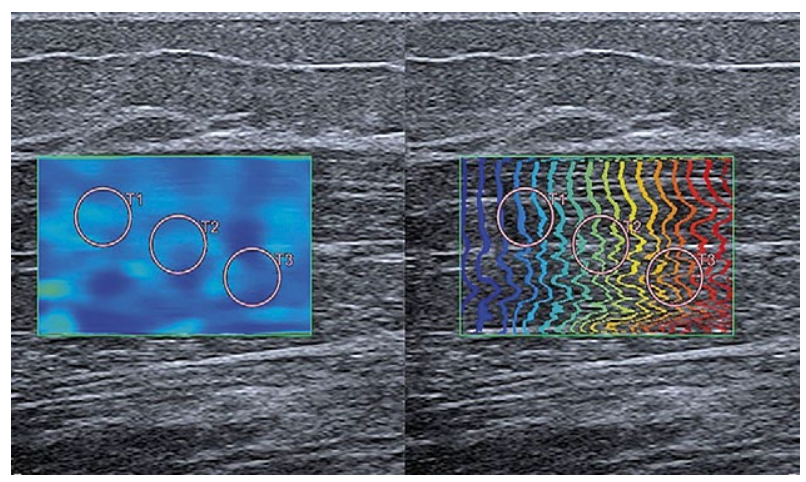

Fig 1. SWE measurements of RF were made using circular ROIs that were placed in the longitudinal view on homogenous muscle parenchyma. 2D-SWE map (left side) and quality mode (right side) are seen.

calculated by the device in percentages to obtain VI (\%) values including the total arterial and venous vascularity supply (fig 2).

\section{Passive static stretching protocols}

The right leg was used as the dominant side in all participants. The dominant leg was determined by determination of the leg that was used most frequently and which felt comfortable in the tourniquets and in the vertical jump that the subject enters while running during offensive rebounds during a basketball game. Two different RF SS exercises were performed. The first movement was knee flexion of the right extremity behind the body while facing forward and standing on the left support leg (fig 3a). The second movement involved the left support leg in 90-degree position while facing forward and touching the kneecap of the right extremity to the ground. The extremity with the knee on the ground had flexion performed by the subject to bring it close to the hip. The stretching durations were determined based on previous studies $[26,27]$. When the participants were able to perform the stretch without discomfort or pain, 30 seconds of stretching was performed (fig $3 b$ ). In group A, two sets of two movements were performed for 30 seconds

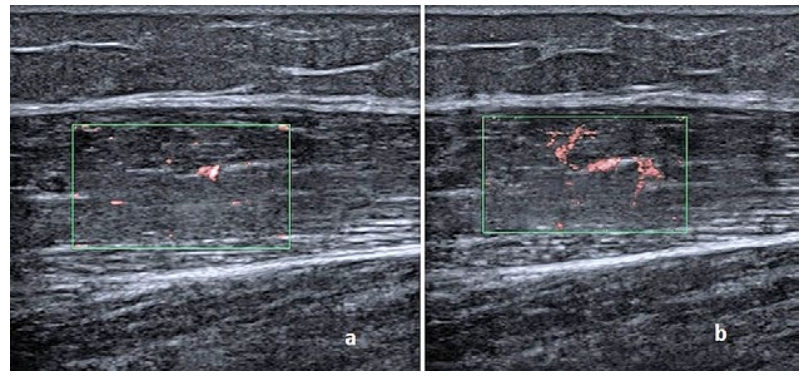

Fig 2. A typical example demonstrating how VI (\%) was obtained on SMI. Variation is observed in VI (\%) in a subject before (a) and after (b) stretching. 


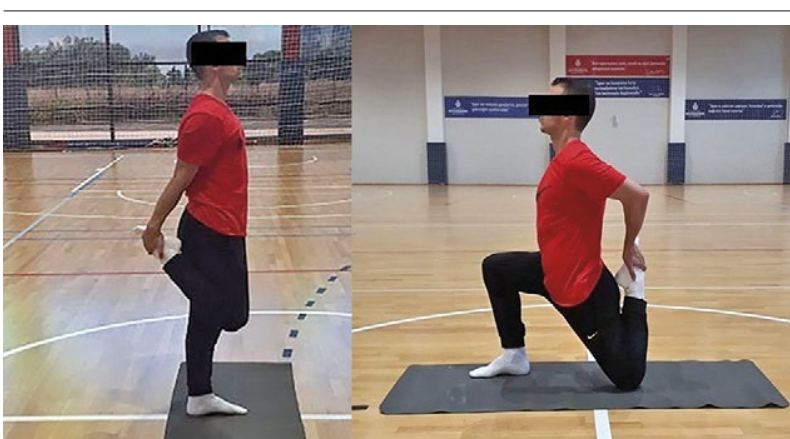

Fig 3. RF muscle stretch methods. $a$. Standing on one leg and pulling the other foot up behind your bottom. $b$. Fully lengthened position for RF with a flexed knee and extended hip by kneeling down.

( $2 \mathrm{~min}$ ), and in group B, the same two movements were performed for five sets of 30 seconds $(5 \mathrm{~min})$. In both groups, 30 seconds of rest were given between the sets.

\section{Statistical Analysis}

Statistical analysis was performed with the SPSS (Statistical Package for the Social Sciences) program. Descriptive statistics of elasticity $(\mathrm{kPa})$, velocity $(\mathrm{m} / \mathrm{s})$, and VI (\%) obtained by two operators at rest, 2 and 5 min of SS were presented as minimum, maximum and median. Normality was tested using the Kolmogorov-Smirnov tests. The visual methods such as histograms and probability plots were also used to determine the normality. The elasticity, velocity and VI values obtained before and after stretching in the groups A and B were compared with the Wilcoxon test for the values without normal distribution. Spearman's correlation coefficient was used to obtain the relationship between stiffness and blood flow parameters and to evaluate the relationship between elasticity and velocity values. $P$ values of less than 0.05 were considered statistically significant. To evaluate the interoperator reliability, we used the Inter-class Correlation Coefficient (ICC) and 95\% confidence interval.

\section{Results}

Descriptive statistics for elasticity, velocity, and VI values obtained by two operators at rest and after 2 and 5 min of SS are presented in Table I. When we analysed all SWE and SMI values from both operators, Spearman's correlation coefficient revealed a statistically significant negative correlation between elasticity and VI parameters $(p=0.011, r=-0.282)$ and between velocity and VI parameters $(\mathrm{p}=0.017, \mathrm{r}=-0.266)$. A significant positive correlation was found between all the elasticity and velocity parameters $(\mathrm{p}<0.001, \mathrm{r}=0.9$; fig 4$)$.

\section{Shear wave elastography}

In group A, there was no significant difference in elasticity and velocity between resting and $2 \mathrm{~min}$ of SS for both operators (all $\mathrm{p}>0.05$ for both operators).

In group B, there was a significant decrease in elasticity and velocity between resting and $5 \mathrm{~min}$ of SS for both operators (first operator $\mathrm{p}=0.009$ and $\mathrm{p}=0.007$, respectively; second operator $\mathrm{p}=0.04$ and $\mathrm{p}=0.03$, respectively).

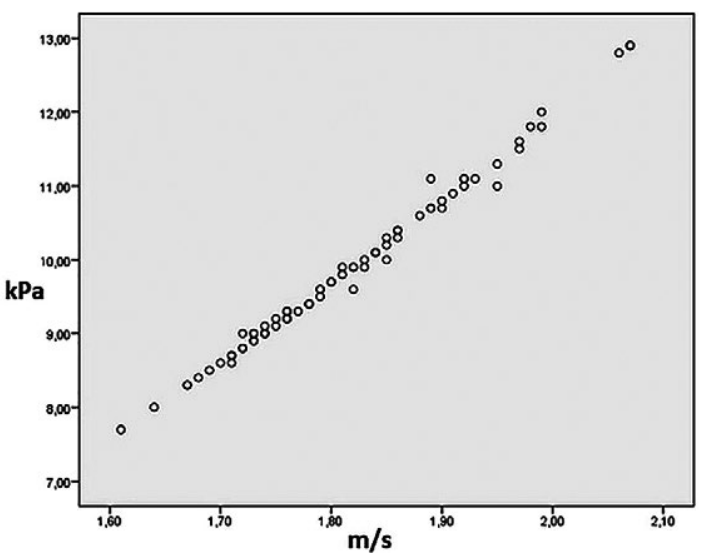

Fig 4. The scatter plot shows a significant positive correlation between the elasticity $(\mathrm{kPa})$ and velocity $(\mathrm{m} / \mathrm{s})$ parameters.
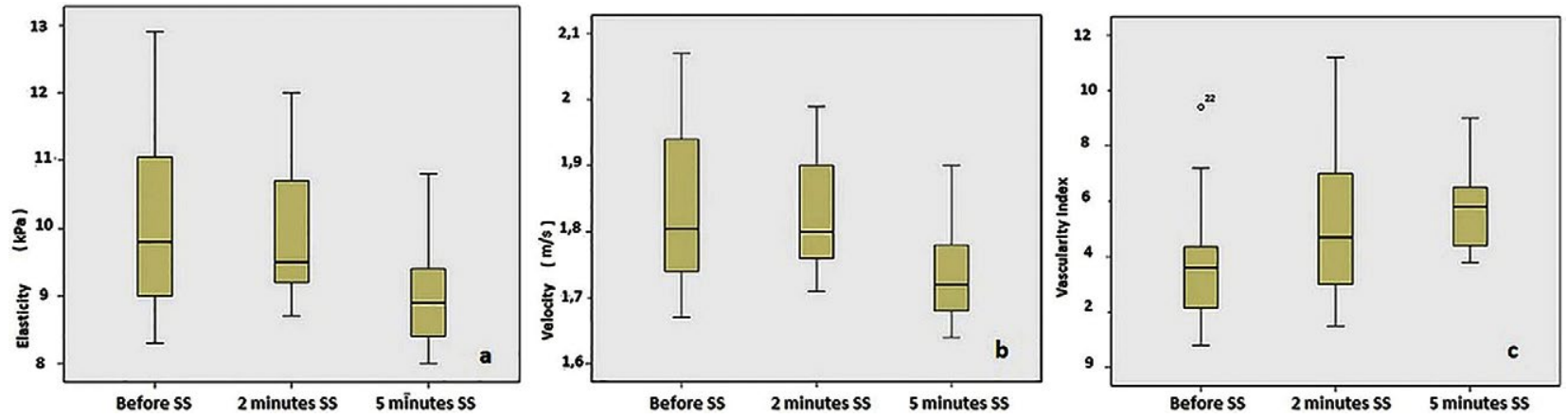

Fig 5. Boxplot showing variation in the median elasticity $(\mathrm{kPa})$, velocity $(\mathrm{m} / \mathrm{s})$, and VI $(\%)$ parameters at rest, and after 2 and $5 \mathrm{~min}$ of SS. There is a minimal decrease in stiffness after 2 min of SS but stiffness was significantly different between rest and at 5 min (a and b). Differences between rest and SS were significant for VI (\%) after both 2 and 5 min of SS (c). 
Table I. Descriptive statistics of elasticity $(\mathrm{kPa})$, Shear Wave velocity $(\mathrm{m} / \mathrm{s})$ and vascularity index $(\%)$ obtained by two operators at rest and after SS and comparison results

\begin{tabular}{|c|c|c|c|c|}
\hline & & $\begin{array}{l}\text { At rest }(\mathbf{n}=\mathbf{1 0}) \\
\text { Median (Min.-Max.) }\end{array}$ & $\begin{array}{l}\text { After SS (n=10) } \\
\text { Median (Min.-Max.) }\end{array}$ & $\mathbf{p}$ \\
\hline \multirow[t]{2}{*}{ Opr. 1} & Elasticity $(\mathrm{kPa})$ & $10.5(8.3-12.9)$ & $9.9(8.7-12)$ & 0.284 \\
\hline & Velocity $(\mathrm{m} / \mathrm{s})$ & $1.87(1.67-2.07)$ & $1.82(1.71-1.99)$ & 0.308 \\
\hline$(0-2 \min )$ & VI $(\%)$ & $2.98(0.8-5.8)$ & $5(1.5-11.2)$ & 0.007 \\
\hline \multirow[t]{2}{*}{ Opr. 1} & Elasticity $(\mathrm{kPa})$ & $9.8(8.6-11.3)$ & $9(8-10.8)$ & 0.009 \\
\hline & Velocity $(\mathrm{m} / \mathrm{s})$ & $1.81(1.71-1.95)$ & $1.73(1.64-1.9)$ & 0.007 \\
\hline$(0-5 \mathrm{~min})$ & VI $(\%)$ & $4.3(2.5-9.4)$ & $5.7(3.8-9)$ & 0.074 \\
\hline \multirow[t]{2}{*}{ Opr. 2} & Elasticity $(\mathrm{kPa})$ & $10.4(8.7-12.8)$ & $9.5(8.5-11.5)$ & 0.180 \\
\hline & Velocity $(\mathrm{m} / \mathrm{s})$ & $1.86(1.71-2.08)$ & $1.82(1.69-1.97)$ & 0.200 \\
\hline$(0-2 \min )$ & VI $(\%)$ & $4.06(0.7-10.8)$ & $6(1.6-14.7)$ & 0.007 \\
\hline \multirow[t]{2}{*}{ Opr. 2} & Elasticity $(\mathrm{kPa})$ & $10.1(9.1-11.8)$ & $9.5(7.7-11.8)$ & 0.044 \\
\hline & Velocity $(\mathrm{m} / \mathrm{s})$ & $1.84(1.75-1.98)$ & $1.76(1.61-1.99)$ & 0.028 \\
\hline$(0-5 \mathrm{~min})$ & VI $(\%)$ & $3.4(1.7-7.4)$ & $5.4(2.5-8.6)$ & 0.005 \\
\hline
\end{tabular}

kPa: KiloPascal; m/s: meters/second; Max: Maximum; Min: Minimum; min.: minutes; n: number of subjects; Opr: Operator; SS: Static Stretching; VI: Vascularity Index

Table II. Inter-operator reliability

\begin{tabular}{|c|c|c|c|c|c|c|c|c|c|c|c|c|}
\hline \multirow[t]{2}{*}{ Variables } & \multicolumn{4}{|c|}{ At rest $(n=20)$} & \multicolumn{4}{|c|}{$2 \min$ of $\operatorname{SS}(n=10)$} & \multicolumn{4}{|c|}{$5 \min$ of $S S(n=10)$} \\
\hline & $\begin{array}{l}\text { ICC } \\
\text { coefficient }\end{array}$ & SEM & $\mathrm{MDC}_{95}$ & $\mathrm{CV}$ & $\begin{array}{l}\text { ICC } \\
\text { coefficient }\end{array}$ & SEM & $\mathrm{MDC}_{95}$ & $\mathrm{CV}$ & $\begin{array}{l}\text { ICC } \\
\text { coefficient }\end{array}$ & SEM & $\mathrm{MDC}_{95}$ & $\mathrm{CV}$ \\
\hline Elasticity $(\mathrm{kPa})$ & 0.81 & 0.087 & 0.241 & 7.1 & 0.62 & 0.129 & 0.357 & 9.2 & 0.52 & 0.159 & 0.440 & 7.8 \\
\hline Velocity (m/s) & 0.79 & 0.008 & 0.022 & 36.3 & 0.57 & 0.012 & 0.033 & 4.5 & 0.54 & 0.014 & 0.039 & 3.8 \\
\hline VI $(\%)$ & 0.82 & 0.165 & 0.457 & 22.9 & 0.77 & 0.395 & 1.094 & 40.8 & 0.83 & 0.146 & 0.404 & 13.4 \\
\hline
\end{tabular}

CV: Coefficient of Variation; ICC: Inter-class Correlation; kPa: KiloPascal; m/s: meters/second; MDC: Minimum Detectable Change; min: minutes; SEM: Standard Error of Measurement; SS: Static Stretching; VI: Vascularity Index

The elasticity and velocity parameters were significantly lower than resting only after 5 min of SS (fig 4).

\section{Superb microvascular imaging}

In group A, there was a significant increase in VI between resting and 2 min of SS for both operators (first operator $\mathrm{p}=0.007$; second operator $\mathrm{p}=0.007$ ).

In group B, there was a significant increase in VI between resting and $5 \mathrm{~min}$ of SS for both operators (first operator $\mathrm{p}=0.007$; second operator $\mathrm{p}=0.005$ ).

The blood flow significantly increased both after 2 and 5 min of SS (fig 5).

\section{Reliability}

Inter-operator reliability was moderate to perfect for measurement of elasticity, velocity and VI at rest and after 2 and 5 min of SS (range: 0.52-0.83; Table II).

\section{Discussions}

To our knowledge, this study was the first to investigate the effects of SS on RF stiffness in adolescents using SWE. Additionally, the originality of the present study comes from the SMI techniques, which have not been evaluated before and which are used to determine the differences before and after muscle stretching. Additionally, this study investigated the inter-operator reliability for SWE and SMI.

In the present study, no significant reduction in RF stiffness after 2 min of SS was observed in adolescents. Maeda et al [4] and Nakamura et al [18] found a significant reduction in gastrocnemius medialis (GM) stiffness after 2 min of SS in adults, which is different from our results. The differences may be a result of investigating different muscles. Different muscles in the body show proportional changes resulting from previous training and frequency of use or function. Another reason may be that our study includes adolescents. Muscles are made from a collection of contractile (e.g., actin and myosin) and noncontractile (e.g., glycogen, water, enzymes) components. Depending on age and hormonal changes, there may be differences in these components. As a result, reactions to SS may be different. Because the similar fundamental stretching techniques and SS durations were used in other similar studies, differences in the protocols between the studies are unlikely to affect the results. Similar to our study, Umehara et al [21] found that the shear elastic modulus of the superior and inferior portions of the infraspinatus decreased significantly after a cross-body stretch (in total $2.5 \mathrm{~min}$ ) with scapular stabilization. 
Studies have been performed using elastography after more than 2 min of SS. In the present study, there was a significant decrease in RF stiffness after 5 min SS in adolescents. Similarly, Nakamura et al [19] and Taniguchi et al [20] and found a decrease in the stiffness of GM and gastrocnemius lateralis (GL) muscles after $5 \mathrm{~min} \mathrm{SS}$ in adults using SWE. Akagi et al [16] found a significant decrease in GM muscle stiffness after 6 min of SS. These results suggest that RF and GM muscles respond with similarly prolonged SS effects. Nordez et al [28], using transient elastography in young adults argued that $10 \mathrm{~min}$ of SS did not reduce GM muscle stiffness. The reason for this difference may be related to the use of different elastography methods and the difference in SS duration.

Responses to the mechanical stimuli of stretch and shear stress play an important role in preserving normal vascular functions. Any disruption in these variables causes a variety of vascular disorders. Hotta et al [12] suggested that acute stretching produces a mechanical lengthening and local ischemia within muscle. Additionally, 4 weeks of daily muscle stretching increases blood flow to skeletal muscles during exercise, enhances endothelium-dependent vasodilatation of resistance arteriole sand increases several morphological indices of $\mathrm{O} 2$ delivery capacity in stretched muscles in old rats. This result investigated the chronic effects of stretching. Studies of animal models found that an increase in muscle length during stretching caused pressure strains in capillaries. Thus, mean capillary diameter and muscle blood flow decrease and vascular resistance increases during stretching [29]. After stretching, mean leg blood flow increases suggesting that there is a post-stretch hyperemic response [26].

This study is the first concerned with acute changes in blood flow in humans after stretching: 2 min of SS seems to be sufficient to increase muscle vascularity. Although there is no clear in vivo information, it would not be surprising that the increased blood flow is useful for the muscle structure. Increased blood flow provides better perfusion, enhances the amount of oxygen and nutrition reaching the muscles, thereby allowing a higher level of physical performance [30]. Increased venous flow may be beneficial to clear the accumulated lactic acid after exercise, whereas increased oxygenation will aid in muscle healing. Thus, the results of the present study may be useful for physical rehabilitation protocols and sports science. For example, in adult chronic stroke or pediatric cerebral palsy, patients who are undergoing rehabilitation of spastic muscles, 5 min of SS stretching may be chosen in rehabilitation protocols instead of $2 \mathrm{~min}$ of SS to ensure optimal softening of the RF. Mathevon et al [31] mentioned that ultrasound associated with SWE shows promise for assessing structural changes in spastic muscles. With some methodological adaptations, that approach could guide spasticity treatment. In addition to the previous data, the present study can make recommendations regarding the optimal time.

In sports sciences, it is widely acknowledged that warming up before vigorous activity is important. The overall goal of any pre-activity routine is to prevent muscle injury and to prepare athletes for practice or competition. The period following warm-up and vigorous physical activity is called the "cool down", or the post-training period. In routine practice, it is widely assumed that cool-down strategies may be used to enhance recovery and reduce muscle injury after training [32]. Warm-up and cool-down stretching is accepted as an adaptation of low intensity exercise. Research indicates that periods of low intensity exercise such as stretching can contribute to long-term positive changes in the elastic properties of the connective tissue within the muscle-tendon architecture [32]. Increasing blood flow provides a greater amount of oxygen to the muscles and better perfusion of muscles allows the possibility of better nutrition. Better intramuscular blood flow provides the opportunity to produce higher levels of physical performance [10]. Despite these well-known benefits of stretching, there are studies proposing that, because SS reduces muscle stiffness, it negatively affects athletic performance [33,34]. Our results show that stiffness parameters did not decrease from resting after $2 \mathrm{~min}$ of SS and that blood flow significantly increased both after 2 and 5 min of SS. As a result, for successful sporting performance, 2 min of SS may be sufficient for the RF in warming up because reducing stiffness negatively affects athletic performance and it may be sufficient for an optimal increase in blood flow. Five minutes of SS may be chosen in cooling down to reduce muscle damage formed during racing, and to help with the recovery process.

Currently, various ultrasound elastography techniques are available. Each technique has advantages and limitations [35,36]. Strain elastography (SE) and acoustic radiation force impulse (ARFI) elastography were the first methods developed for this purpose. However, the overall use of SWE has increased in recent years because it is easy to apply, more operator-independent and provides quantitative results. SMI is a novel Doppler method, and its greatest advantage is that it shows very fine vascular structures compared to color Doppler and power Doppler [22]. The present study supports this information and the inter-operator reliability indicates significant correlation for SWE and SMI. They may reliably be used for immobile and surface organs such as muscles, especially in physical rehabilitation and training sciences. Similarly, 
Jeon et al found that the inter-observer reliability for ARFI was excellent for ankle-plantar flexion with submaximal isometric contraction $(\mathrm{ICC}=0.968)$ and good for the relaxed position (ICC $=0.891$ ) [13]. Alfuraih et al studied the combination of medium ROI and longitudinal orientation from the lateral location for SWE resulted in a strong internal agreement of intra-class correlation of $0.76(0.57-0.89)$ for the new system and an almost perfect agreement of $0.92(0.82-0.97)$ for the established system [15]. Inter-system reproducibility for the best combination was $0.71(0.48-1)$ with a mean velocity of $0.07 \pm 0.49 \mathrm{~m} / \mathrm{s}$.

There are several limitations to our study. Firstly, the study cohort could be expanded to include a greater number of participants of both genders. Another limitation is that subjects performed SS under their own control and determined the limit of stretch based on their own pain threshold. A dynamometer was not used to ensure more objective stretching. Finally, acute effects were researched with no investigation into the effects of stretching durations in the chronic period on muscle stiffness.

In conclusion, SWE and SMI can be used to acquire reliable quantitative data on muscle stiffness and blood flow in adolescents. In medical physical rehabilitation protocols, 5 min of SS may be chosen to reduce RF stiffness and to prevent cramps, spasms, and muscle damage. Additionally, because reducing stiffness negatively affects athletic performance, 2 min of SS may be sufficient for an optimal increase in blood flow during warm-up procedures. Five min of SS may enhance recovery and reduce injury during cool-down protocols after training.

Acknowledgements: We would like to acknowledge Mrs. Manolya Kara for assisting with the review of the publication and general academic editing.

\section{Conflict of interest: none}

\section{References}

1. Page P. Current concepts in muscle stretching for exercise and rehabilitation. Int J Sports Phys Ther 2012;7:109-119.

2. 2.Thompson AJ, Jarrett L, Lockley L, Marsden J, Stevenson VL. Clinical management of spasticity. J Neurol Neurosurg Psychiatry 2005;76:459-463.

3. Ayala F, De Ste Croix M, Sainz De Baranda P, Santonja F. Acute effects of static and dynamic stretching on hamstring eccentric isokinetic strength and unilateral hamstring to quadriceps strength ratios. J Sports Sci 2013;31:831-839.

4. Maeda N, Urabe Y, Tsutsumi S, et al. The Acute Effects of Static and Cyclic Stretching on Muscle Stiffness and Hardness of Medial Gastrocnemius Muscle. J Sports Sci Med 2017;16:514-520.
5. Wilson GJ, Elliott BC, Wood GA. Stretch shorten cycle performance enhancement through flexibility training. Med Sci Sports Exerc 1992;24:116-123.

6. Witvrouw E, Danneels L, Asselman P, D'Have T, Cambier D. Muscle flexibility as a risk factor for developing muscle injuries in male professional soccer players. A prospective study. Am J Sports Med 2003;31:41-46.

7. Ruan M, Zhang Q, Wu X. Acute Effects of Static Stretching of Hamstring on Performance and Anterior Cruciate Ligament Injury Risk During Stop-Jump and Cutting Tasks in Female Athletes. J Strength Cond Res 2017;31:1241-1250.

8. Sá MA, Neto GR, Costa PB, et al. Acute Effects of Different Stretching Techniques on the Number of Repetitions in A Single Lower Body Resistance Training Session. J Hum Kinet 2015;45:177-185.

9. Kuebler WM, Uhlig U, Goldmann T, et al. Stretch activates nitric oxide production in pulmonary vascular endothelial cells in situ. Am J Respir Crit Care Med 2003;168:13911398.

10. Bassett DR Jr, Howley ET. Limiting factors for maximum oxygen uptake and determinants of endurance performance. Med Sci Sports Exerc 2000;32:70-84.

11. Arpino JM, Nong Z, Li F, et al. Four-Dimensional Microvascular Analysis Reveals That Regenerative Angiogenesis in Ischemic Muscle Produces a Flawed Microcirculation. Circ Res 2017;120:1453-1465.

12. Hotta K, Behnke BJ, Arjmandi B, et al. Daily muscle stretching enhances blood flow, endothelial function, capillarity, vascular volume and connectivity in aged skeletal muscle. J Physiol 2018;596:1903-1917.

13. Jeon M, Youn K, Yang S. Reliability and quantification of gastrocnemius elasticity at relaxing and at submaximal contracted condition. Med Ultrason 2018;20:342-347.

14. Akkoc O, Caliskan E, Bayramoglu Z. Effects of passive muscle stiffness measured by Shear Wave Elastography, muscle thickness, and body mass index on athletic performance in adolescent female basketball players. Med Ultrason 2018;20:170-176.

15. Alfuraih AM, O'Connor P, Tan AL, Hensor E, Emery P, Wakefield RJ. An investigation into the variability between different shear wave elastography systems in muscle. Med Ultrason 2017;19:392-400.

16. Akagi R, Takahashi H. Acute effect of static stretching on hardness of the gastrocnemius muscle. Med Sci Sports Exerc 2013;45:1348-1354.

17. Hirata K, Miyamoto-Mikami E, Kanehisa H, Miyamoto N. Muscle-specific acute changes in passive stiffness of human triceps surae after stretching. Eur J Appl Physiol 2016;116:911-918.

18. Nakamura M, Ikezoe T, Kobayashi T, et al. Acute effects of static stretching on muscle hardness of the medial gastrocnemius muscle belly in humans: an ultrasonic shear-wave elastography study. Ultrasound Med Biol 2014;40:19911997.

19. Nakamura M, Ikezoe T, Nishishita S, Umehara J, Kimura M, Ichihashi N. Acute effects of static stretching on the shear elastic moduli of the medial and lateral gastrocnemius 
muscles in young and elderly women. Musculoskelet Sci Pract 2017;32:98-103.

20. Taniguchi K, Shinohara M, Nozaki S, Katayose M. Acute decrease in the stiffness of resting muscle belly due to static stretching. Scand J Med Sci Sports 2015;25:32-40.

21. Umehara J, Hasegawa S, Nakamura M, et al. Effect of scapular stabilization during cross-body stretch on the hardness of infraspinatus, teres minor, and deltoid muscles: An ultrasonic shear wave elastography study. Musculoskelet Sci Pract 2017;27:91-96.

22. Machado P, Segal S, Lyshchik A, Forsberg F. A Novel Microvascular Flow Technique: Initial Results in Thyroids. Ultrasound Q 2016;32:67-74.

23. Kruse NT, Silette CR, Scheuermann BW. Influence of passive stretch on muscle blood flow, oxygenation and central cardiovascular responses in healthy young males. Am J Physiol Heart Circ Physiol 2016;310:1210-1221.

24. Chino K, Kawakami Y, Takahashi H. Tissue elasticity of in vivo skeletal muscles measured in the transverse and longitudinal planes using shear wave elastography. Clin Physiol Funct Imaging 2017;37:394-399.

25. Gennisson JL, Deffieux T, Mace E, Montaldo G, Fink M, Tanter M. Viscoelastic and anisotropic mechanical properties of in vivo muscle tissue assessed by supersonic shear imaging. Ultrasound Med Biol 2010;36:789-801.

26. Ryan ED, Beck TW, Herda TJ, et al. The time course of musculotendinous stiffness responses following different durations of passive stretching. J Orthop Sports Phys Ther 2008;38:632-639.

27. Bandy WD, Irion JM. The effect of time on static stretch on the flexibility of the hamstring muscles. Phys Ther 1994;74:845-850.
28. Nordez A, Gennisson JL, Casari P, Catheline S, Cornu C. Characterization of muscle belly elastic properties during passive stretching using transient elastography. J Biomech 2008;41:2305-2311.

29. Poole DC, Mathieu-Costello O. Capillary and fiber geometry in rat diaphragm perfusion fixed in situ at different sarcomere lengths. J Appl Physiol 1992;73:151-159.

30. Bassett DR Jr, Howley ET. Limiting factors for maximum oxygen uptake and determinants of endurance performance. Med Sci Sports Exerc 2000;32:70-84.

31. Mathevon L, Michel F, Aubry S, et al. Two-dimensional and shear wave elastography ultrasound: A reliable method to analyse spastic muscles? Muscle Nerve 2018;57:222228.

32. Popp JK, Bellar DM, Hoover DL, et al. Pre- and Post-Activity Stretching Practices of Collegiate Athletic Trainers in the United States. J Strength Cond Res 2017;31:2347-2354.

33. Bradley PS, Olsen PD, Portas MD. The effect of static, ballistic, and proprioceptive neuromuscular facilitation stretching on vertical jump performance. J Strength Cond Res 2007;21:223-226.

34. Peck E, Chomko G, Gaz DV, Farrell AM. The effects of stretching on performance. Curr Sports Med Rep 2014;13:179-185.

35. Bamber J, Cosgrove D, Dietrich CF, et al. EFSUMB guidelines and recommendations on the clinical use of ultrasound elastography. Part 1: Basic principles and technology. U1traschall Med 2013;34:169-184.

36. Cosgrove D, Piscaglia F, Bamber J, et al. EFSUMB guidelines and recommendations on the clinical use of ultrasound elastography. Part 2: Clinical applications. Ultraschall Med 2013;34:238-253. 